\title{
Self-consistent $\boldsymbol{\beta}$ functions and emittances of round colliding beams
}

\author{
A. V. Otboyev and E. A. Perevedentsev \\ Budker Institute of Nuclear Physics, Novosibirsk, 630090, Russia \\ (Received 20 May 1999; published 18 October 1999)
}

\begin{abstract}
The flip-flop effect with the linearized beam-beam force is formulated through self-consistent $\beta$ functions and equilibrium emittances which are both affected by collision. We give the results of two models of emittance dependence. The effect of finite bunch length is also discussed.
\end{abstract}

PACS numbers: 29.27.Bd, 29.27.Fh

\section{INTRODUCTION}

From many observations of the beam-beam effects on existing $e^{+} e^{-}$colliders, it is known that under some conditions the sizes of opposing bunches become very different. This phenomenon is called the flip-flop effect. Such a state is not stable and the bunches may exchange their sizes. The flip-flop effect leads to reduction of the luminosity because of the difference in bunch sizes resulting in reduction of the effective interaction area.

The problem is greatly simplified by linearization of the beam-beam force; it has been studied in terms of evolution of the second moments of the beam distribution, involving the radiation effects: damping and quantum excitation [1-3].

Another way to understand this problem is formulation in terms of self-consistent dynamic $\beta$ functions of colliding beams at the interaction point (IP) [4]. The equilibrium emittances of the bunches are affected by the linear part of the beam-beam force: action of the opposing bunch is roughly equivalent to insertion of a (thin) lens modifying the arc lattice [5]. So, a correct account for these dynamic emittance variations should be done in a selfconsistent way.

This paper gives results of the self-consistent model for round colliding beams, and calculation of the equilibrium radiation emittance with the thin lens insertion. We also discuss a simple model representing the bunch length effect.

\section{SELF-CONSISTENT $\beta$ FUNCTIONS}

We adopt here the conditions of round colliding beams [6]: equal $\beta$ functions $\beta_{x}^{*}=\beta_{y}^{*}$, equal tunes $\nu_{x}=\nu_{y}$, and equal emittances $\epsilon_{x}=\epsilon_{y}$ in the nominal optics of the collider.

Consider a collider lattice with one IP and the betatron phase advance on the arc $\mu_{0}=2 \pi \nu$. The betatron oscillations in the arc are described by their matrix $M_{0}$,

$$
M_{0}=\left(\begin{array}{cc}
\cos \mu_{0} & \beta^{*} \sin \mu_{0} \\
-\frac{\sin \mu_{0}}{\beta^{*}} & \cos \mu_{0}
\end{array}\right) .
$$

We take, for simplicity, the Twiss parameter $\alpha_{0}=0$ at the IP. The opposite bunch is represented in the linear approximation by the thin lens matrix

$$
F=\left(\begin{array}{cc}
1 & 0 \\
-\frac{4 \pi \xi}{\beta^{*}} & 1
\end{array}\right) .
$$

The beam-beam parameter $\xi$ involves the sizes of this bunch and reads as

$$
\begin{aligned}
\xi_{x, y} & =\frac{N r_{0} \beta^{*}}{2 \pi \gamma \sigma_{x, y}\left(\sigma_{x}+\sigma_{y}\right)} \\
& =\frac{2 \xi_{0}}{e_{x, y} \sqrt{\beta_{x, y} / \beta^{*}}\left(\sqrt{\beta_{x} / \beta^{*}}+\sqrt{\beta_{y} / \beta^{*}}\right)} .
\end{aligned}
$$

In the last equality, $\xi_{0}=N r_{0} /\left(4 \pi \gamma \epsilon_{0}\right)$ is the nominal beam-beam parameter for round beams, and $e_{x, z}=$ $\epsilon_{x, y} / \epsilon_{0}$ are normalized emittances of the bunch. Then we can get the resulting matrix of one revolution $M=F M_{0}$. From $M$ we obtain new values of $\mu$ and the $\beta$ function, modified by collision. For both horizontal and vertical planes, the appropriate equations are

$$
\begin{gathered}
\cos \mu_{1,2}=\cos \mu_{0}-2 \pi \xi_{2,1} \sin \mu_{0}, \\
\beta_{1,2}=\beta^{*} \frac{\sin \mu_{0}}{\sin \mu_{1,2}},
\end{gathered}
$$

where subscripts 1,2 indicate colliding bunches. Let us consider equal intensities of the colliding bunches (equal $\xi_{0}$ ). At first it is convenient to transform Eq. (5) into the form

$$
\left(\frac{\beta^{*}}{\beta_{1,2}}\right)^{2}=\frac{1-\cos ^{2} \mu_{1,2}}{\sin ^{2} \mu_{0}} .
$$

Substituting Eq. (4) into the last equation, using Eq. (3), after some calculations [4] we obtain a set of equations on self-consistent $\beta$ functions in the special case of round beams,

$$
\begin{aligned}
& b_{1}^{2}=1+2 x c \frac{b_{2}}{e_{2}}-x^{2} \frac{b_{2}^{2}}{e_{2}^{2}}, \\
& b_{2}^{2}=1+2 x c \frac{b_{1}}{e_{1}}-x^{2} \frac{b_{1}^{2}}{e_{1}^{2}} .
\end{aligned}
$$

Here we used a convenient notation: $x=2 \pi \xi_{0}, c=$ $\cot 2 \pi \nu, b_{i}=\beta^{*} / \beta_{i}$. The problem is periodic in $\nu$ with 
the period $1 / 2$; therefore, we consider only $0<\nu<1 / 2$ in the following.

\section{CONSTANT EMITTANCES}

We start with the case of constant emittances $e_{1}=$ $e_{2}=1$. The system (7) has equal,

$$
b_{1}=b_{2}=\frac{x c+\sqrt{1+x^{2}\left(1+c^{2}\right)}}{1+x^{2}},
$$

and unequal solutions,

$$
=\frac{x c \sqrt{1+x^{2}} \pm \sqrt{1-\left(2+3 c^{2}\right) x^{2}+\left(1+c^{2}\right) x^{4}}}{\left(x^{2}-1\right) \sqrt{1+x^{2}}} .
$$

Unequal solutions of Eqs. (7) $b_{i}$ correspond to the flipflop situation. They are real and positive when $\nu \in$ $(0,1 / 4)$ and $x$ belongs to the interval

$$
\sqrt{\frac{2+3 c^{2}+c \sqrt{8+9 c^{2}}}{2\left(1+c^{2}\right)}}<x<c+\sqrt{1+c^{2}} \text {. }
$$

For $\nu \in(1 / 4,1 / 2)$, the inequalities should be reversed. Small $\nu$ are of predominant interest for a high beam-beam performance. One can obtain the threshold value of $x$ for small $\nu$ by taking the limit $c \rightarrow \infty$ in the left-hand side of Eq. (10): $x_{\mathrm{th}}=\sqrt{3}$. This is a very large and unrealistic value of $x$, which corresponds to $\xi_{0} \simeq 0.26$.

Another way to get $x_{\text {th }}$ is the graphical method [7], applied to Eqs. (7): we consider $b_{1}$ as a function of $b_{2}$, and evaluate the derivative $\partial b_{1} / \partial b_{2}$ at the point of equal $b_{i}$, thus testing the possibility for unequal solutions to appear. Then the flip-flop threshold $x=x_{\text {th }}$ satisfies the equation

$$
\left.\frac{\partial b_{1}}{\partial b_{2}}\right|_{b 1=b 2}=-1,
$$

yielding the same $x_{\text {th }}$ as in the left-hand side of Eq. (10).

In contrast to the above solution, one may expect a nonround final flip-flop state, e.g., a cross-shaped one,

$$
b_{1 x}=b_{2 y}=b_{x} \equiv w_{x}^{2}, \quad b_{1 y}=b_{2 x}=b_{y} \equiv w_{y}^{2} .
$$

The system (7) now takes the form

$$
\begin{aligned}
& w_{x}^{4}=1+\frac{4 x c w_{y}^{2} w_{x}}{w_{x}+w_{y}}-\frac{4 x^{2} w_{y}^{4} w_{x}^{2}}{\left(w_{x}+w_{y}\right)^{2}}, \\
& w_{y}^{4}=1+\frac{4 x c w_{x}^{2} w_{y}}{w_{x}+w_{y}}-\frac{4 x^{2} w_{x}^{4} w_{y}^{2}}{\left(w_{x}+w_{y}\right)^{2}} .
\end{aligned}
$$

Applying the graphical method to the system (12) (see Fig. 1) and using Eq. (11) we obtain this threshold:

$$
x \geq x_{\text {th }}=\sqrt{\frac{4+5 c^{2}+c \sqrt{24+25 c^{2}}}{2\left(1+c^{2}\right)}} .
$$

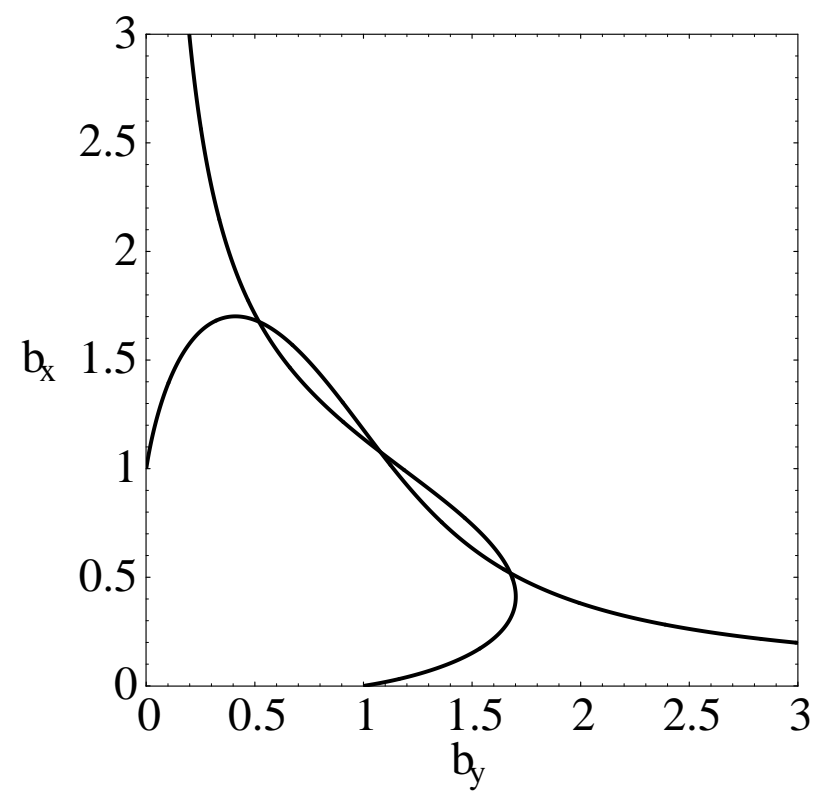

FIG. 1. Graphical method for solving Eqs. (12) on selfconsistent $\beta$ functions. Intersection on the primary diagonal corresponds to the equal solutions, additional intersections describe the flip-flop solutions.

It appears to be even higher than that for the round flip-flop state: the round beam shape seems to be "flipproof"; cf. Ref. [8]. Figure 2 shows all threshold curves of the flip-flop state appearance in the case of constant emittances of colliding bunches.

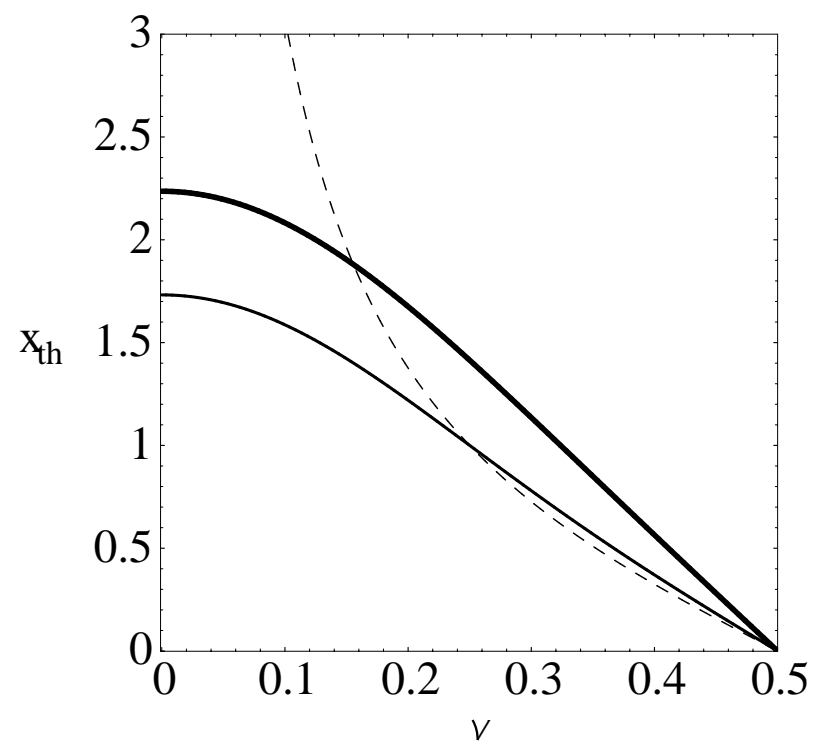

FIG. 2. The flip-flop border curves. The thick solid curve corresponds to the case of "crosswise flip," when the vertical size of one bunch in the final state is equal to the horizontal size of its partner and vice versa. The dashed line presents the right-hand side of Eq. (10). 


\section{RADIATION EMITTANCE}

Assuming the emittances unchanged by collision in the above section, we see that the flip-flop thresholds are rather high in terms of $\xi_{0}$. However, in $e^{+} e^{-}$colliders we need to consider radiation emittances as affected by the linearized beam-beam force ${ }^{1}$. An approach that takes radiation into account is given in Refs. [1,2], where the authors work in terms of second moments of the bunch distribution. The radiation damping effect is included in the damping matrix $\operatorname{diag}\left(1, \lambda, \lambda^{2}\right)$ in [1] and $\operatorname{diag}(\lambda, \lambda, \lambda)$ in [2], $\lambda$ is the damping coefficient. The quantum excitation is represented by the diffusion vector, which is proportional to $\left(0,0,1-\lambda^{2}\right)$ in [1], and $(1-\lambda, 0,1-\lambda)$ in [2], and inserted at the IP. Then the authors construct the one-turn map for the second moments, find its fixed points, and calculate the equilibrium emittances.

However, equations on the bunch sizes and the values of modified emittances are dependent on the treatment of radiation. For example, starting from the quantum excitation vector in the form $\left(0,0,1-\lambda^{2}\right)$ as in [1] with the appropriate damping matrix, the authors have the equilibrium dynamic emittance proportional to the dynamic $\beta$ function of the bunch (i.e., $e_{i}=1 / b_{i}$ in normalized variables). Because of this, the flat beam case in [1] corresponds to our round beam case with constant emittance, Eq. (7), while the round beam case in [1] leads to equations

$$
\begin{aligned}
& b_{1}^{2}=1+2 x c b_{2}^{2}-x^{2} b_{2}^{4}, \\
& b_{2}^{2}=1+2 x c b_{1}^{2}-x^{2} b_{1}^{4} .
\end{aligned}
$$

In [2] the radiation effect is taken from the diffusion equation (the Fokker-Planck equation for the phasespace particle distribution), where only momentum is considered as fluctuating. With this assumption for the radiation model, the authors of Ref. [2] require that the radiation damping matrix and excitation vector should have the symmetric form $\operatorname{diag}(\lambda, \lambda, \lambda)$ and $(1-\lambda, 0,1-$ $\lambda)$. With such a treatment of radiation, it is obtained in [2] that the equilibrium radiation emittance with one additional lens at the IP always exceeds the nominal one.
In our opinion, the diffusion vector in the form of Refs. [1,2] cannot take into account the radiation action distributed around all the bends of the machine arcs; the value of the modified radiation emittance may either decrease or increase with the strength of the inserted lens, depending on the arc lattice.

Let us prove the above statement by exact evaluation of the equilibrium radiation emittance in the lattice with a thin lens inserted at the IP, to represent the linear beambeam effect. Aiming at the first order in radiation effects, it is more efficient to proceed in terms of the radiation integrals, rather than to use the method of Refs. [1,2] with appropriate corrections in the diffusion vector.

In what follows, zero dispersion at the IP is assumed for simplicity, and so the dispersion in the arc $\eta(s)$ is left unchanged by the insertion lens; therefore, among all the radiation integrals [9] only $I_{5}$ is modified due to dynamic $\beta$ beat.

The equilibrium emittance is determined by the oneturn average of the Courant-Snyder quadratic form with the dispersion function (cf. [10])

$$
H(s)=\gamma(s) \eta(s)^{2}+2 \alpha(s) \eta(s) \eta^{\prime}(s)+\beta(s) \eta^{\prime}(s)^{2} .
$$

The lens insertion modifies the Twiss parameters involved in $H(s)$, thus changing the radiation emittance. In the Floquet parametrization $H(s)=|W(s)|^{2}$, the appropriate Wronskian reads

$$
W(s)=\left|\begin{array}{cc}
\eta(s) & w(s) \\
\eta^{\prime}(s) & w^{\prime}(s)+i / w(s)
\end{array}\right| e^{i \phi(s)},
$$

where $w=\beta^{1 / 2}$ is the absolute value of the complex Floquet function [11], and $\phi$ is its phase. The modified Floquet function $w(s) e^{\mathrm{i} \phi(s)}$ should be decomposed via the basis of two unperturbed Floquet vectors at the IP, then propagated through the unperturbed arc to get the modified vector on the current azimuth of integration $s$.

The transport matrix $T(f \mid i)$ through the arc can be expressed in terms of initial and final Twiss parameters of unperturbed optics $w_{i}$ and $w_{f}$, respectively,

$$
T(f \mid i)=\left(\begin{array}{cc}
\frac{w_{f}}{w_{i}}\left(\cos \phi-w_{i} w_{i}^{\prime} \sin \phi\right) & w_{f} w_{i} \sin \phi \\
\left(\frac{w_{f}^{\prime}}{w_{i}}-\frac{w_{i}^{\prime}}{w_{f}}\right) \cos \phi-\left(w_{f}^{\prime} w_{i}^{\prime}+\frac{1}{w_{f} w_{i}}\right) \sin \phi & \frac{w_{i}}{w_{f}}\left(\cos \phi+w_{f} w_{f}^{\prime} \sin \phi\right)
\end{array}\right),
$$

where $i, f$ denote the initial and final azimuths. Then mapping of the Floquet vectors from the IP to the azimuth $s$ with the phase advance of $\phi$ has the form

$$
T(f \mid i)\left(\begin{array}{c}
w_{i} \\
w_{i}^{\prime}+\frac{i}{w_{i}}
\end{array}\right)=\left(\begin{array}{c}
w_{f} \\
w_{f}^{\prime}+\frac{i}{w_{f}}
\end{array}\right) e^{i \phi} .
$$

\footnotetext{
${ }^{1}$ The presented model leaves out nonlinear beam-beam effects.
}

From the matrix of one revolution with the lens at the IP, we obtain the new value of the initial Floquet vector modified by the insertion, expressed via the unperturbed one,

$$
\begin{aligned}
w & =w_{i} \sqrt{\sin \mu / \sin \mu_{0}}, \\
w^{\prime} & =\left(w_{i}^{\prime}-k w_{i}\right) \sqrt{\sin \mu / \sin \mu_{0}}, \\
\cos \mu & =\cos \mu_{0}-k w_{i}^{2} \sin \mu_{0} .
\end{aligned}
$$


Here $\mu_{0}=2 \pi \nu$ is the betatron phase advance of the unperturbed lattice, $\mu$ is its value modified by the insertion lens, and $2 k$ is the strength of the lens, no assumptions are needed on its smallness. For further calculations we use the notation $\sqrt{\sin \mu / \sin \mu_{0}}=r$, while $p=k w_{i}^{2}$ stands for the normalized strength of the lens.

The modified Floquet vector at the initial point (past the insertion) given by Eq. (15) is then decomposed via the unperturbed basis at the IP,

$$
\left(\begin{array}{c}
w \\
w^{\prime}+\frac{i}{w}
\end{array}\right)=\frac{r^{2}+1+i p r^{2}}{2 r}\left(\begin{array}{c}
w_{i} \\
w_{i}^{\prime}+\frac{i}{w_{i}}
\end{array}\right)+\frac{r^{2}-1-i p r^{2}}{2 r}\left(\begin{array}{c}
w_{i} \\
w_{i}^{\prime}-\frac{i}{w_{i}}
\end{array}\right) .
$$

Now we propagate the new Floquet vector through the arc, using Eqs. (14) to express the modified final vector via the basis of unperturbed ones on the current azimuth $s$. Thus, the modified value of the Wronskian on the azimuth of integration is

$$
W=\frac{1}{2}(r+1 / r+i p r) W_{0}(s)+\frac{1}{2}(r-1 / r-i p r) W_{0}^{*}(s) .
$$

Finally, finding from the above equation the new value of the dispersion invariant $H=|W|^{2}$, we obtain for the linear beam-beam effect on the integrand of the radiation integral $I_{5}$

$$
\frac{H(s)}{H_{0}(s)}=\frac{1+p \cot 2 \pi \nu}{\sqrt{1+2 p \cot 2 \pi \nu-p^{2}}}+\frac{p \csc 2 \pi \nu \cos 2\left(\arg W_{0}(s)-\pi \nu\right)}{\sqrt{1+2 p \cot 2 \pi \nu-p^{2}}},
$$

where the 0 subscript marks the quantities relevant to the unperturbed lattice.

The relation (17) is an exact result of the linear lattice theory, conventionally taking radiation effects into account to first order. The $1+p \cot 2 \pi \nu$ term in (17) gives positive definite contribution $\propto 1+p^{2}$ to the radiation emittance, and compares to the result of [5]. But the second term, proportional to $\cos 2\left(\arg W_{0}(s)-\pi \nu\right)$, depends on the arc lattice, and generally its contribution $\propto p$ to the radiation integral $I_{5}$ does not vanish. It may well override the effect of the first term in some particular lattices, resulting in a linear slope of either sign in the emittance dependence on $\xi_{0}$ (see Fig. 3), contradicting [2,5].

\section{MODELS OF EMITTANCE VARIATION}

We can implement the above conclusion in simple models of variable emittance, to be used jointly with Eqs. (7) for the self-consistent analysis.

The first model assumes the linear variation of emittance with the strength of the lens of the opposite bunch: we have then for the normalized emittances

$$
e_{1}=1+k x \frac{b_{2}}{e_{2}}, \quad e_{2}=1+k x \frac{b_{1}}{e_{1}},
$$

where $k$ is the linear slope coefficient and should be kept not too large for our model to be valid. We solve Eqs. (18) for $e_{1}$ and $e_{2}$ first, then substitute these solutions into Eqs. (7) to obtain two equations on the two variables $\left(b_{1}, b_{2}\right)$,

$$
\begin{aligned}
& b_{1}^{2}=1+\frac{4 b_{2} x\left[c\left(1+\left(b_{1}-k x\right)+\sqrt{D}\right)-b_{2} x\right]}{\left(1+\left(b_{1}-b_{2}\right) k x+\sqrt{D}\right)^{2}}, \\
& b_{2}^{2}=1+\frac{4 b_{1} x\left[c\left(1+\left(b_{2}-b_{1}\right) k x+\sqrt{D}\right)-b_{1} x\right]}{\left(1+\left(b_{2}-b_{1}\right) k x+\sqrt{D}\right)^{2}},
\end{aligned}
$$

where $D=4 b_{2} k x+\left(1+\left(b_{1}-b_{2}\right) k x\right)^{2}$.
Now because of the very complicated form of the last system, we may solve the problem using method [7]. With $k>0$, the flip-flop situation appears only at high values of $x=2 \pi \xi_{0}$; Fig. 4 shows such a dependence. In this case the values of the self-consistent $\beta$ functions are small enough and the emittances exceed their nominal values.

The case $k<0$ is more interesting. Equal solutions of Eqs. (19) may be expressed in the form

$$
b=\frac{\alpha^{2}-1}{4 k x},
$$

where $\alpha$ is a positive real root of the equation

$$
\begin{aligned}
\alpha^{4}+2\left(2 x^{2}-1\right) \alpha^{2}-8 x^{2}(1+2 c k) \alpha+ \\
1+4 x^{2}\left(1+4 c k-4 k^{2}\right)=0 .
\end{aligned}
$$

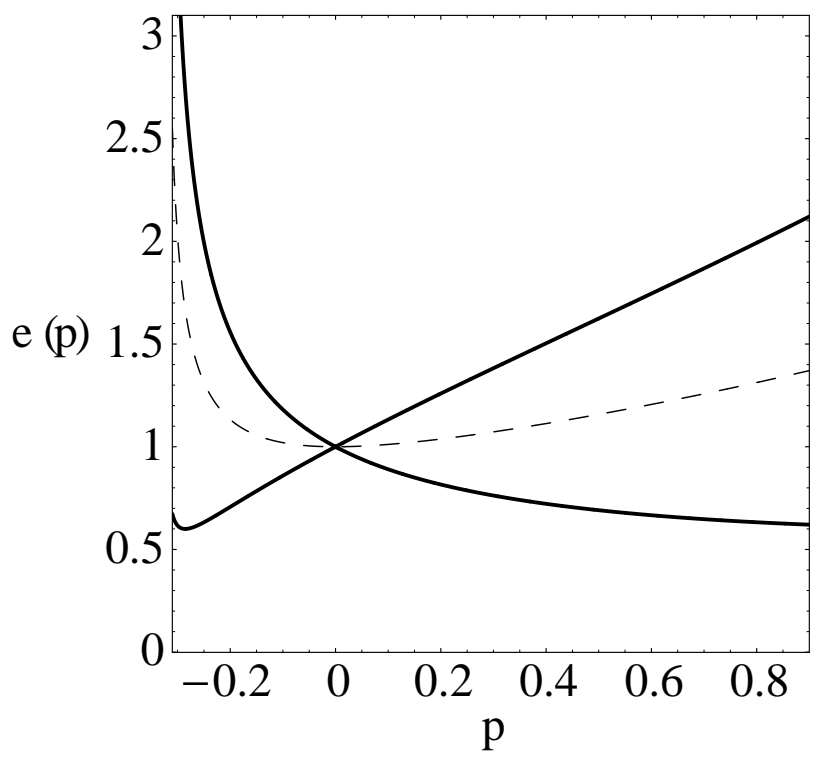

FIG. 3. Equilibrium radiation emittance as a function of the insertion lens. Either sign of the slope may appear depending on the arc lattice. The dashed line corresponds to the result obtained in $[2,5]$. 


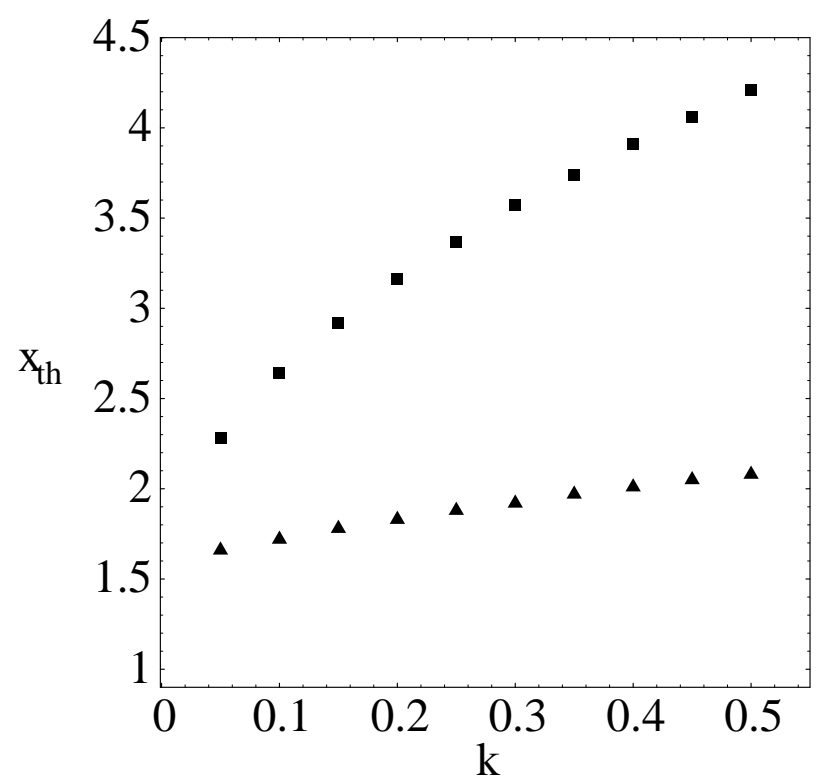

FIG. 4. The flip-flop threshold $x_{\mathrm{th}}$ vs the positive slope $k$ in (19), $\nu=0.01$ (top), $\nu=0.1$ (bottom).

The requirement for $b$ to be real and positive leads to conditions on $\alpha$ of

$$
0<\alpha<1 .
$$

In terms of beam intensity it means there is some limiting value of $x$, which depends on the values of $k$ and $\nu$,

$$
x<\frac{1}{2 \sqrt{4 k^{2}-4 c k-1}} .
$$

If $x$ is above this limit, the system (19) has no equal solutions. Before $x$ approaches its maximal value, Eqs. (19) have one solution with equal $b_{i}$. Unequal (i.e., flip-flop) solutions may be represented as two roots of a quadratic equation

$$
y^{2}+p y+q=0
$$

with $b_{1}+b_{2}=-p, b_{1} b_{2}=q$, and $p, q$ expressed in terms of Eqs. (19). Then after finding $p, q$, we require their reality $\left[x_{0}(k)\right.$ shown with a thin solid curve in Fig. 5] and $p^{2}-4 q$ (the appropriate discriminant) to be positive [ $x_{1}(k)$ is the thick curve in Fig. 5]; the threshold value for the flip-flop state appearance is determined as $x_{\mathrm{th}}=$ $\max \left(x_{1}, x_{0}\right)$. Unequal (flip-flop) solutions appear at high values of the beam-beam parameter, and $x_{\text {th }}$ increases with the shift of $k$ in the negative direction. We also observed that both the limiting value for equal solutions and $x_{\text {th }}$ for the flip-flop state appearance (see Fig. 5) grow with the increase of the tune $\nu$.

In the second model we assume linear variation of the beam sizes with the strength of the lens of the opposite bunch. After some calculations, this model is expressed

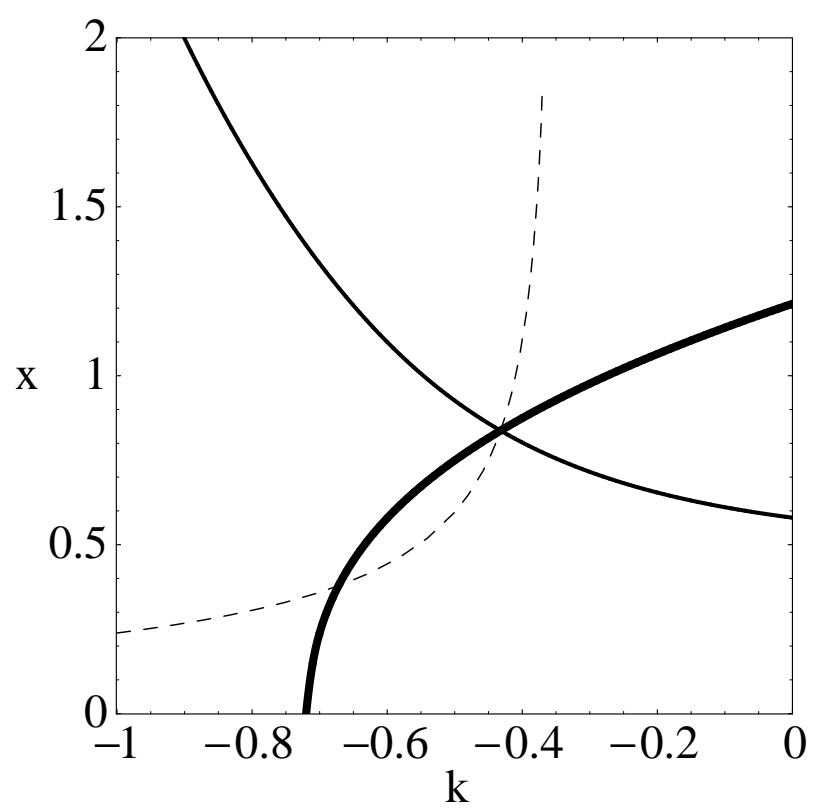

FIG. 5. Limiting curves of solutions in the model of linear variation of beam emittances with the lens of the opposite bunch. Normal solutions exist under the dashed line. Unequal $b_{1}, b_{2}$ appear for all values of $k$ above the upper of the two solid curves.

by the equations

$$
e_{1}=b_{1}\left(1+k x \frac{b_{2}}{e_{2}}\right)^{2}, \quad e_{2}=b_{2}\left(1+k x \frac{b_{1}}{e_{1}}\right)^{2},
$$

and it is reducible to two variables $e_{1} / b_{1}$ and $e_{2} / b_{2}$ only. Hence, we solve Eqs. (20) for these and substitute into Eqs. (7) to obtain solutions for $b_{i}$.

The resultant of two equations in Eqs. (20) has simple factorization

$$
\begin{aligned}
R= & \left(e_{2}^{2}+2 b_{2} e_{2} k x+b_{2}^{2} k^{2} x^{2}-b_{2} e_{2} k^{2} x^{2}\right) \\
& \times\left(e_{2}^{3}-b_{2} e_{2}^{2}-2 b_{2}^{2} e_{2} k x-b_{2}^{3} k^{2} x^{2}\right) .
\end{aligned}
$$

The first factor gives two solutions:

$$
e_{2}=\frac{1}{2} b_{2} k x(k x-2 \pm \sqrt{k x(k x-4)}) .
$$

So, for $k>0$ the flip-flop threshold is high: we need $k x>4$ for $e_{1,2}$ to be real. The second factor in $R$ has one real root, if $k>0$. It corresponds to the normal solution $b_{1}=b_{2}$.

Another situation is the case of $k<0$. Now the roots of Eq. (21) are always real and positive and correspond to the flip-flop solutions. After substituting the solution of Eq. (21) into Eqs. (7) we get $b_{1,2}^{2}$ and require that they be positive; this yields the condition on the existence of the flip-flop solutions

$$
\begin{array}{r}
x<x(k)=\tan \pi \nu(\cot \pi \nu+1 / k)^{2}, \\
\tan \pi \nu<-k .
\end{array}
$$




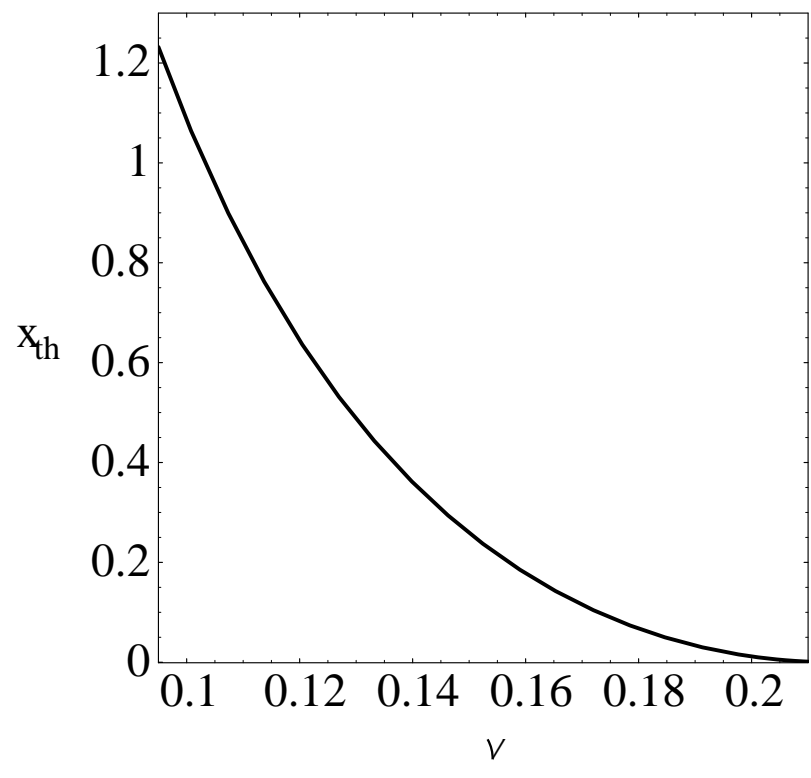

FIG. 6. The flip-flop threshold $x_{\text {th }}$ vs the tune $\nu$ in the case of $k<0$ in (20), $k=-0.8$. The flip-flop area lies under the curve.

These expressions indicate how to avoid the unwanted flip-flop situation: at some $x$ and a given value of $k$ in the linear dependence of emittances, we may raise the tune to shift it in the area of only equal solutions, crossing the flip-flop border shown in Fig. 6.

\section{EFFECT OF THE BUNCH LENGTH}

In this section we present the constant emittance model, accounting for the effect of bunch length in collision by splitting either of the colliding bunches into two equal infinitely short bunches spaced by $l$ (in units of $\beta_{0}$ ) (Fig. 7).

The interaction process then has three phases: (i) collision of particles 2 and 3 at the IP; (ii) collision of 2 and 1 , and collision of 3 and 4 , at the points positioned at the distance of $\mp l / 2$ from the IP; (iii) collision of particles 1 and 4 at the IP.

Figure 8 shows the behavior of the $\beta$ function of one particle, when it collides with its counter partners, and their "lenses" at points 0 and $l / 2$ change this $\beta$ function.

All values of the Twiss parameters $\alpha$ and $\beta$ for each particle are taken at the IP. Therefore we need to recalculate them to the side points. It is convenient to make this with the appropriate Steffen matrix of the drift space and one additional lens

$$
T_{3}=\left(\begin{array}{ccc}
(l p-1)^{2} & 2 l(l p-1) & l^{2} \\
p(1-l p) & (1-2 l p) & -l \\
p^{2} & 2 p & 1
\end{array}\right)
$$

Here $p$ is the normalized strength of the lens. With this matrix, $T_{3}$, we get the strength of lens 3 in the point of

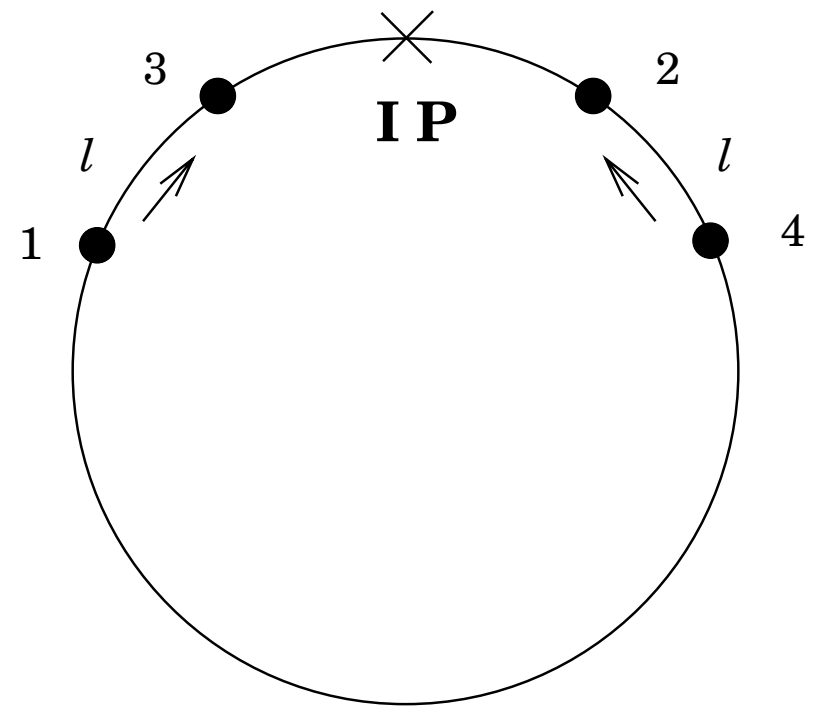

FIG. 7. The model of the bunch length effect. Each bunch consists of two particles with constant distance $l$ between them.

interaction with 4 ,

$$
p_{34}=\frac{x}{\frac{\left(1+\alpha_{3}^{2}\right) l^{2}}{4 \beta_{3}}+\beta_{3}\left(1-\frac{l x}{2 \beta_{2}}\right)^{2}+\alpha_{3} l\left(-1+\frac{l x}{2 \beta_{2}}\right)} .
$$

In the last formula, $\beta_{3}$ and $\alpha_{3}$ are the Twiss parameters of the third particle at the IP and so on. The same technique gives

$$
p_{21}=\frac{x}{\frac{\left(1+\alpha_{2}^{2}\right) l^{2}}{4 \beta_{2}}+\beta_{2}\left(1-\frac{l x}{2 \beta_{3}}\right)^{2}+\alpha_{2} l\left(-1+\frac{l x}{2 \beta_{3}}\right)} .
$$
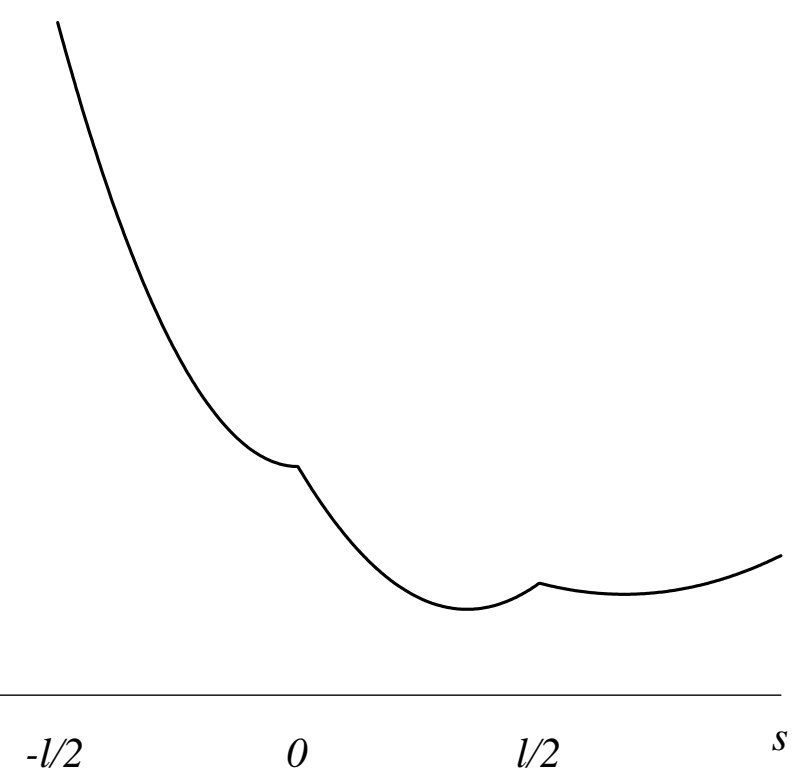

FIG. 8. Dependence of the $\beta$ function of one particle on the azimuth along the arc. The counter particles act as lenses at points 0 and $l / 2$. 


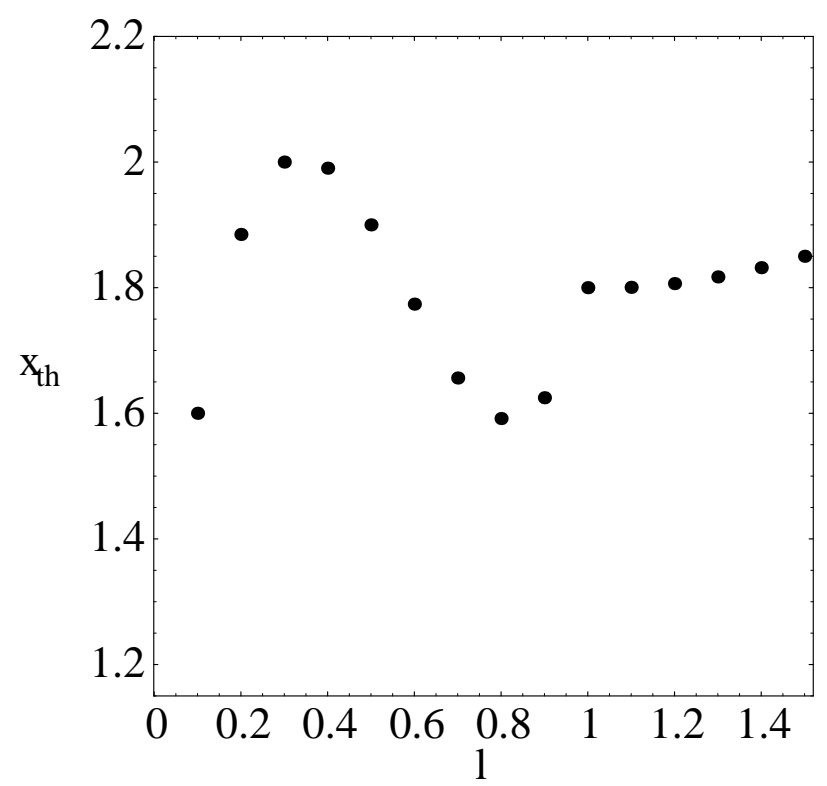

FIG. 9. The flip-flop threshold $x_{\text {th }}$ vs $l$ in the model of the bunch length.

For particles 1 and 4 we have the conditions

$$
T_{3}\left(l / 2, p_{34}\right)\left(\beta_{43}, \alpha_{43}, \gamma_{43}\right)=\left(\beta_{4}, \alpha_{4}, \gamma_{4}\right) .
$$

The last equation yields

$$
p_{43}=\frac{4 \beta_{4} x}{4 \beta_{4}^{2}+4 \alpha_{4} \beta_{4} l+\left(1+\alpha_{4}^{2}\right) l^{2}},
$$

and by analogy

$$
p_{12}=\frac{4 \beta_{1} x}{4 \beta_{1}^{2}+4 \alpha_{1} \beta_{1} l+\left(1+\alpha_{1}^{2}\right) l^{2}} .
$$

Now we can write the resulting matrix of one revolution for the particle, say 1 ,

$$
M_{1}=M_{0} M_{l}(l / 2) M_{p}\left(p_{21}\right) M_{l}(-l / 2) M_{p}\left(x / \beta_{4}\right),
$$

and for the other particles their matrices can be written similarly. From the matrix of one revolution for each particle $M_{i}(i=1, \ldots, 4)$ we get the new values of the phase advance $\mu, \beta$, and $\alpha$ functions, and then obtain the equations on the self-consistent $\beta$ functions. This system is very complicated and can be studied only numerically. The first conclusion is that if $l \neq 0$ there is no situation, when all $\beta$ functions are equal. We have the state in our system, when parameters of the front and back particles are equal. We define the flip-flop situation when all four parameters are different; the threshold for these solutions to appear is high (see Fig. 9). Therefore we conclude that the finite bunch length effect is not detrimental in the round beam case.

\section{CONCLUSION}

The flip-flop effect is studied in terms of the selfconsistent $\beta$ function in the case when the emittances of colliding bunches are influenced by the linear part of the beam-beam force. Evaluation of the radiation emittance of the bunch from the radiation integral is presented in the case of a thin lens inserted at the IP, with the emphasis on the term omitted in [5].

We have presented two models of variable emittances. One of them, when the emittance has a linear dependence on the strength of the lens of the opposite bunch, gives high flip-flop thresholds in the area of positive slope $k$ in Eqs. (18) and gives no threshold but the colliding bunches are of equal size if $k<0$ and the beam intensity is below a certain limit. Unequal solutions (i.e., the flip-flop state) in this case appear at high values of the beam-beam parameter and the value of $x_{\text {th }}$ is even higher than the limit of normal solution existence.

The second model (20) predicts low flip-flop thresholds only when we assume $k<0$, i.e., the size of the bunch is decreased by the force of the opposite lens. We can avoid the flip-flop situation here by the working point maneuver. However, lattices with $k>0$ seem to be generally preferable against the flip-flop effect.

The influence of the bunch length on the flip-flop effect thresholds in our simple model is weak.

\section{ACKNOWLEDGMENTS}

We acknowledge useful discussions of the subject with P. M. Ivanov, I. A. Koop, I. N. Nesterenko, and D. V. Pestrikov.

[1] M. A. Furman, K. Y. Ng, and A.W. Chao, SSC Report No. SSC-174, 1988.

[2] K. Hirata and F. Ruggiero, Part. Accel. 28, 137-142 (1990).

[3] S. Matsumoto and K. Hirata, KEK Report No. 91-12, 1992.

[4] D. V. Pestrikov, http://www.gsi.de/struck/contrib.html; GSI Report No. 97-07, GSI, 1997.

[5] K. Hirata and F. Ruggiero, LEP Note 611, 1988.

[6] V. V. Danilov et al., in Proceedings of the 5th European Particle Accelerator Conference, Barcelona, Spain, 1996 (Institute of Physics, Bristol, UK, 1996), Vol 2, p. 1149.

[7] A. B. Temnykh, INP Report No. INP 82-148, 1982.

[8] D. V. Pestrikov, KEK Report No. 98-230, 1999.

[9] R.H. Helm et al., IEEE Trans. Nucl. Sci. NS-20, 900 (1973).

[10] M. Sands, SLAC Report No. 121, 1970.

[11] A. A. Kolomensky and A. N. Lebedev, Theory of Cyclic Accelerators (North-Holland, Amsterdam, 1966). 